\title{
STUDY ON THE EFFECT OF STREPTOKINASE-ACTIVATED PLASMIN (FIBRINOLYSIN) ON CLOTS IN VARIOUS STAGES OF ORGANIZATION ${ }^{1,2}$
}

\author{
By NATHAN BACK, JULIAN L. AMBRUS, C. LENORE SIMPSON, AND \\ SIDNEY SHULMAN
}

(Irom The Roswell Park Memorial Institute and the Departments of Pharmacology, Bacteriology and Immunology, and Pathology, Unizersity of Buffalo Medical School, Buffalo, N. Y.)

(Submitted for publication July 25, 1956; accepted February 20, 1958)

In previous studies $(1,4)$, a method was developed for the quantitative recording of the lysis of thrombi and emboli produced with I $^{131}$-labeled fibrinogen. Using this method, the in vivo fibrinolytic activity of human and bovine plasmin was demonstrated (2). Other proteolytic enzymes (crude pancreatic protease, trypsin, carboxypeptidase, papain and ficin) were ineffective in maximal tolerated doses $(1,3,4)$. Literature on the therapeutic use of proteolytic enzymes has been reviewed $(1,2)$. In all previous experiments, treatment was started one-half hour after forming labeled clots in the experimental animals. The question arose whether plasmin treatment would be effective in dissolving organized clots and whether there is a correlation between degree of organization and plasmin-induced lysis. The preparations referred to as "plasmin" in this study actually contain a variety of enzymatic activities; they are fibrinolytic, proteolytic and are able to activate the fibrinolysin system. Presumably they represent a mixture of plasmin, plasmin-activator and free streptokinase. It is difficult to ascribe therapeutic activity to any one of these components. The purpose of these experiments is to study therapeutic indications and conditions for the preparations currently undergoing clinical trial. Labeled clots were produced in several veins of dogs at various time intervals. At the time of treatment, these clots were of various ages. Differences in lysis were established by recording

1 Preliminary report presented before the Federation of American Societies of Experimental Biology, Atlantic City, 1956 (Fed. Proc. 1956, 15, 396).

2 Supported by grants from The American Heart Association, Parke Davis and Co., The Sharp and Dohme Division of Merck and Co., and the United States Public Health Service. disappearance of radioactivity and by histopathologic study.

\section{MATERIALS AND METHODS}

Materials and methods were similar to those described in previous studies (1). Under aseptic conditions, $\mathrm{I}^{131}$-labeled purified bovine fibrinogen was introduced through side branches into isolated segments of veins of dogs and clotted with the aid of thrombin. Semi-constricting aluminum wire ligatures were placed proximal to the clots and artery clamps isolating the segment were released one-half hour after clot formation. Radioactivity over the area was recorded with the aid of a specially constructed lead shield, a scintillation counter, an analytical radiation rate meter, and an Esterline recorder.

Mongrel dogs, 10 to $16 \mathrm{Kg}$. in body weight, were used. At the time of the beginning of treatment, the clots varied in age from 30 minutes to 10 days. In most dogs two clots of the same age were present; one was removed before institution of plasmin treatment. All other clots were removed after the last treatment. Sections from at least two areas of each clot were examined histologically.

Five daily intravenous doses of human plasmin, 30 plasmin units (Loomis) per Kg., ${ }^{3}$ were given. One mg. of the plasmin preparation used was found to contain 3 fibrinolytic plasmin units (Loomis) or 0.098 proteolytic casein unit (6). The specific activity was 0.28 casein unit per mg. protein. This dose was previously found (2) to cause lysis of fresh clots but not to alter fibrinogen and prothrombin levels. In most previous studies the dose was administered in four hour infusions; in the present experiments, rapid intravenous injections were given. In the previous studies, human plasmin was prepared from Cohn's Fraction III according to the method of Kline (7). In the present study a simple streptokinaseactivated acid extract of Fraction III was used, prepared by Dr. Werner Baumgarten, Sharp and Dohme Division of Merck and Co., West Point, Pa. The following information was made available on the method of preparation of this material. Fraction III, prepared by the

${ }^{3}$ A plasmin unit (Loomis) is defined as the quantity of enzyme needed to dissolve $0.6 \mathrm{ml}$. of a 0.3 per cent purified bovine fibrin clot at $45^{\circ} \mathrm{C}$. in two minutes at $\mathrm{pH}$ $7.2(5)$. 
alcohol fractionation method of Cohn, was extracted with acid, adjusted to $\mathrm{pH} 7.8$, and to each equivalent of $40 \mathrm{mg}$. of Fraction III, 13,000 Christensen units of streptokinase was added (U. S. Patent $2,666,729$ ). We found that when lyophilized samples of the above material were diluted with increasing volumes of physiological saline, fibrinolytic activity first increased, then decreased. Such bellshaped dilution versus activity curves could be duplicated by adding excessive amounts of streptokinase to plasminogen prepared by the Kline method. This was interpreted as indicating that the preparation used in this study contained a considerable amount of free streptokinase. It is known that most batches of streptokinase are poor activators of dog plasminogen. On the other hand Sherry, Titchener, Gottesman, Wasserman, and Troll have shown (8) that streptokinase-activated human plasmin preparations may activate dog plasminogen. It is presently a matter of controversy whether this effect of streptokinaseactivated human plasmin is due to the presence of "activator" (9), or to the activating ability of a hypothetical plasminogen-streptokinase complex $(10,11)$, or a plasminstreptokinase complex $(4,11)$.
Blood samples were taken before, and at various time intervals after, plasmin injections for hematological and biochemical analyses as in previous studies (1-4). Prothrombin time is reported as clotting index: the ratio of the prothrombin time prior to treatment to the prothrombin time during and after plasmin administration. In most experiments as in previous studies (1-4) prothrombin time was also determined after adding bovine fibrinogen, so as to eliminate the effect of fibrinogenopenia on prothrombin time determinations.

\section{Clot lysis}

Figure 1 shows an experiment in which a dog with one-half hour, one, two and three day old clots was treated with daily injections of 30 plasmin units (Loomis) per $\mathrm{Kg}$. of human plasmin. The first point on the graph indicates radioactivity over the thrombus at the time of clot formation; the second represents radioactivity immediately

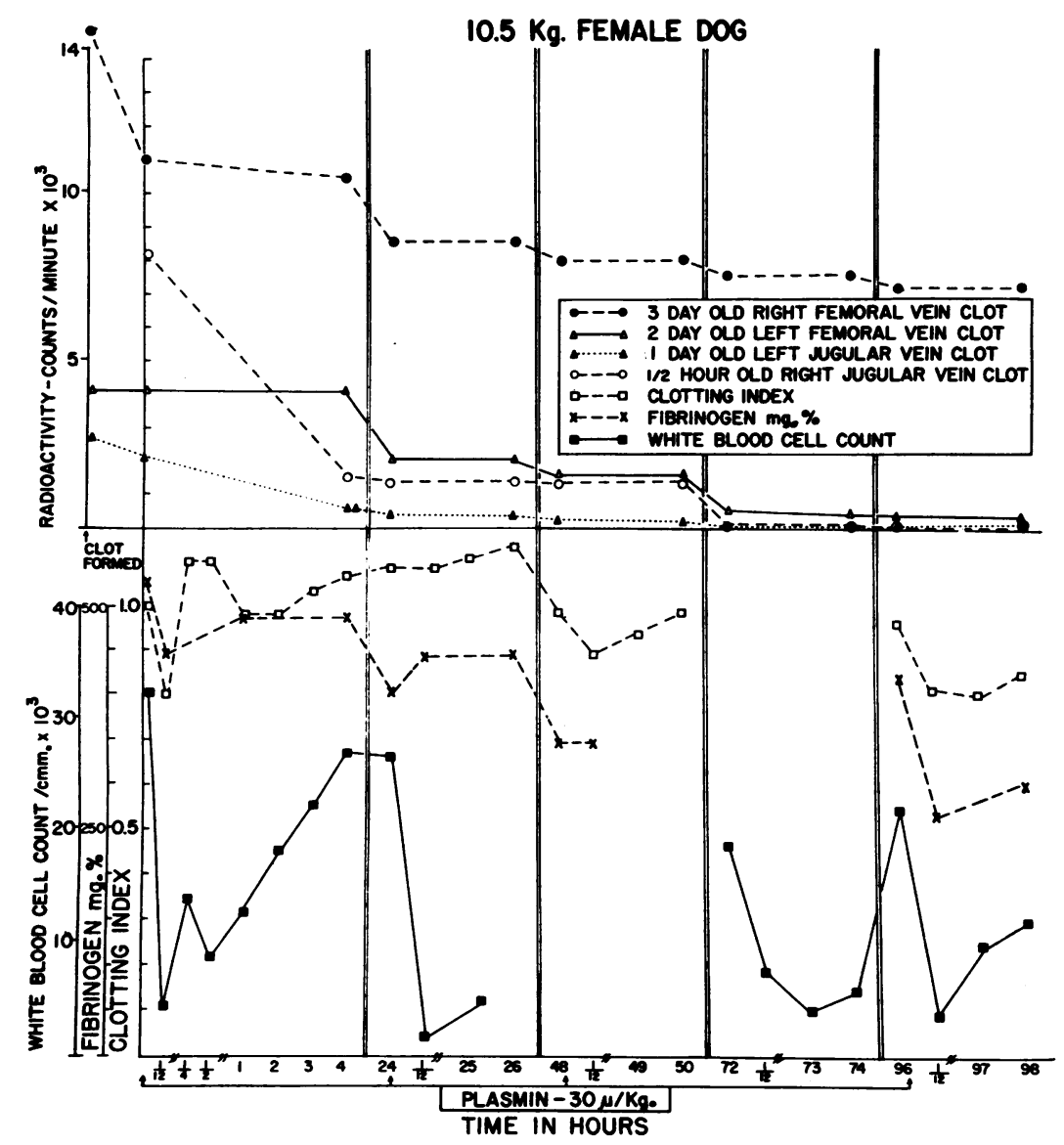

Fig. 1. Effect of Plasmin on One-Half Hour, One, Two and Three Day Old Clots, Clotting Index, Fibrinogen Level and White Blood Cell Count 


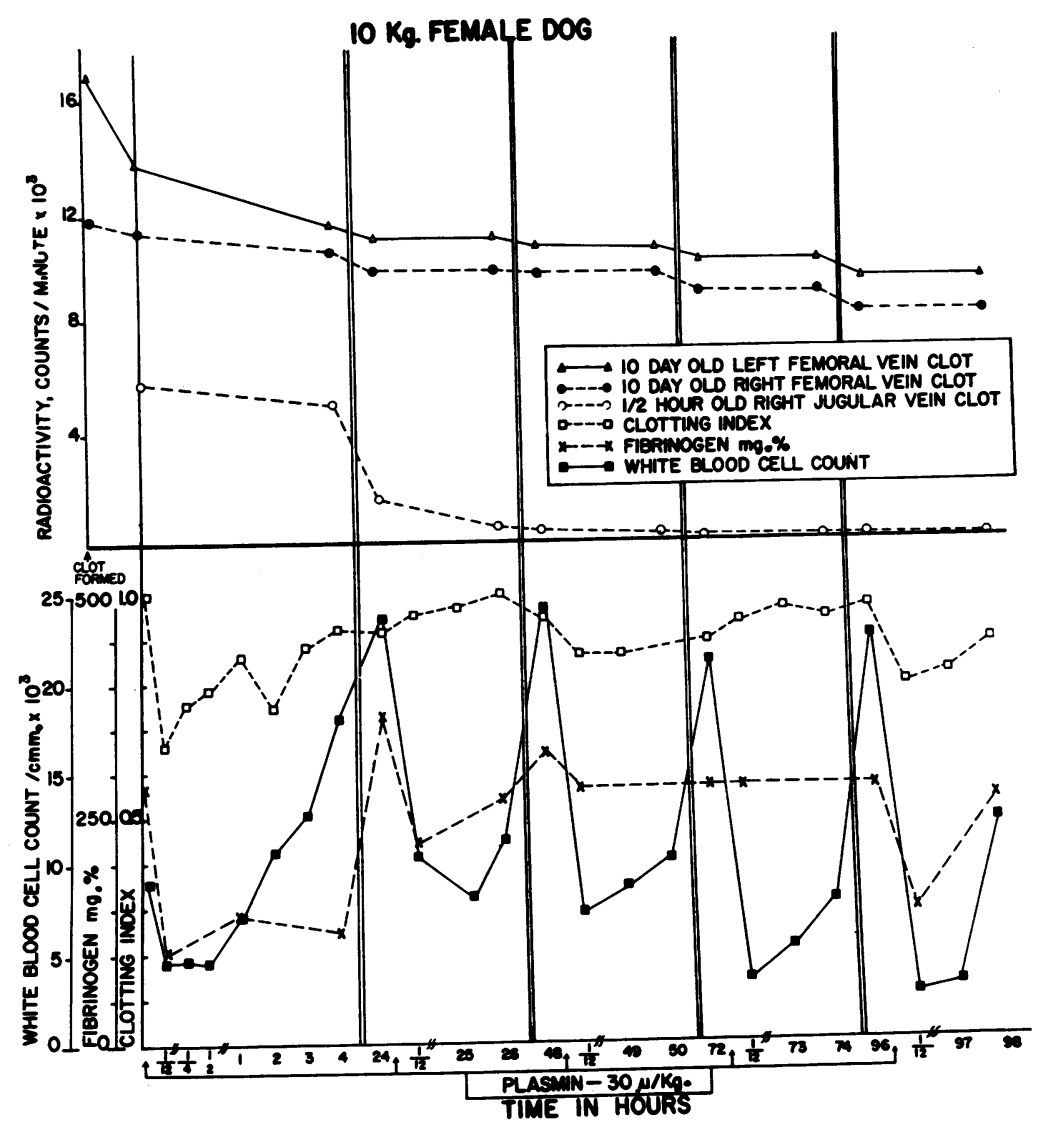

Fig. 2. Effect of Plasmin on One-Half Hour and 10 Day Old Clots, Clotting Index, Fibrinogen Level and White Blood Cell Count

before initiation of treatment. It can be seen that ${ }^{131}$-labeled clots up to the age of two days completely disappeared during the five day treatment period. Only slight lysis was evidenced in the

TABLE I

Effect of clot age on lysis

\begin{tabular}{|c|c|c|c|}
\hline $\begin{array}{c}\text { Age of } \\
\text { clots }\end{array}$ & $\begin{array}{l}\text { No. of } \\
\text { clots }\end{array}$ & $\begin{array}{c}\text { Treatment } \\
\text { of dogs }\end{array}$ & $\begin{array}{l}\text { Mean of \% lysis } \\
\text { and standard error } \\
\text { of mean at end of } \\
\text { treatment period }\end{array}$ \\
\hline$\frac{1}{2} \mathrm{hr}$ & 23 & $\begin{array}{c}\text { saline } \\
\text { (control) }\end{array}$ & $23.54 \pm 3.17$ \\
\hline $\begin{array}{l}10 \text { days } \\
5 \text { days } \\
3 \text { days } \\
2 \text { days } \\
1 \text { day } \\
\frac{1}{2} \text { hr. }\end{array}$ & $\begin{array}{c}4^{*} \\
4 \\
10 \\
6 \\
6 \\
6 \dagger\end{array}$ & $\begin{array}{l}30 \text { plasmin } \\
\text { units (Loomis) } \\
\text { per Kg. body } \\
\text { weight }\end{array}$ & $\begin{array}{l}23.72 \pm 3.75 \\
22.43 \pm 2.63 \\
20.49 \pm 3.55 \\
80.17 \pm 4.26 \\
80.53 \pm 4.79 \\
97.42 \pm 1.10\end{array}$ \\
\hline
\end{tabular}

* Eight additional clots gave similar results but are not included since somewhat different methods were used.

$\dagger$ Three additional clots gave similar results after treatment with more purified plasmin preparations. three day clot. It should be remembered, however, that statistical analysis of lysis of all three day old clots versus control clots revealed no significant difference. Figure 2 illustrates an experiment in which a dog with two 10 day old clots and one-half hour old clot was treated. Radioactivity over the half-hour old clot disappeared as in the previous experiment, but the 10 day old clots showed no significant lysis compared to clots in untreated dogs (2). Results with five day old clots were identical with those of 10 day old clots. Fresh I ${ }^{131}$-labeled fibrinogen was prepared before each series of experiments. Thus, clots of various ages produced in the same dog had various starting levels of radioactivity. No relationship was found between base radioactivity levels and degree of lysis.

Table I summarizes the results of all clots investigated in this study. It appears that under the conditions of these experiments and at the dose 


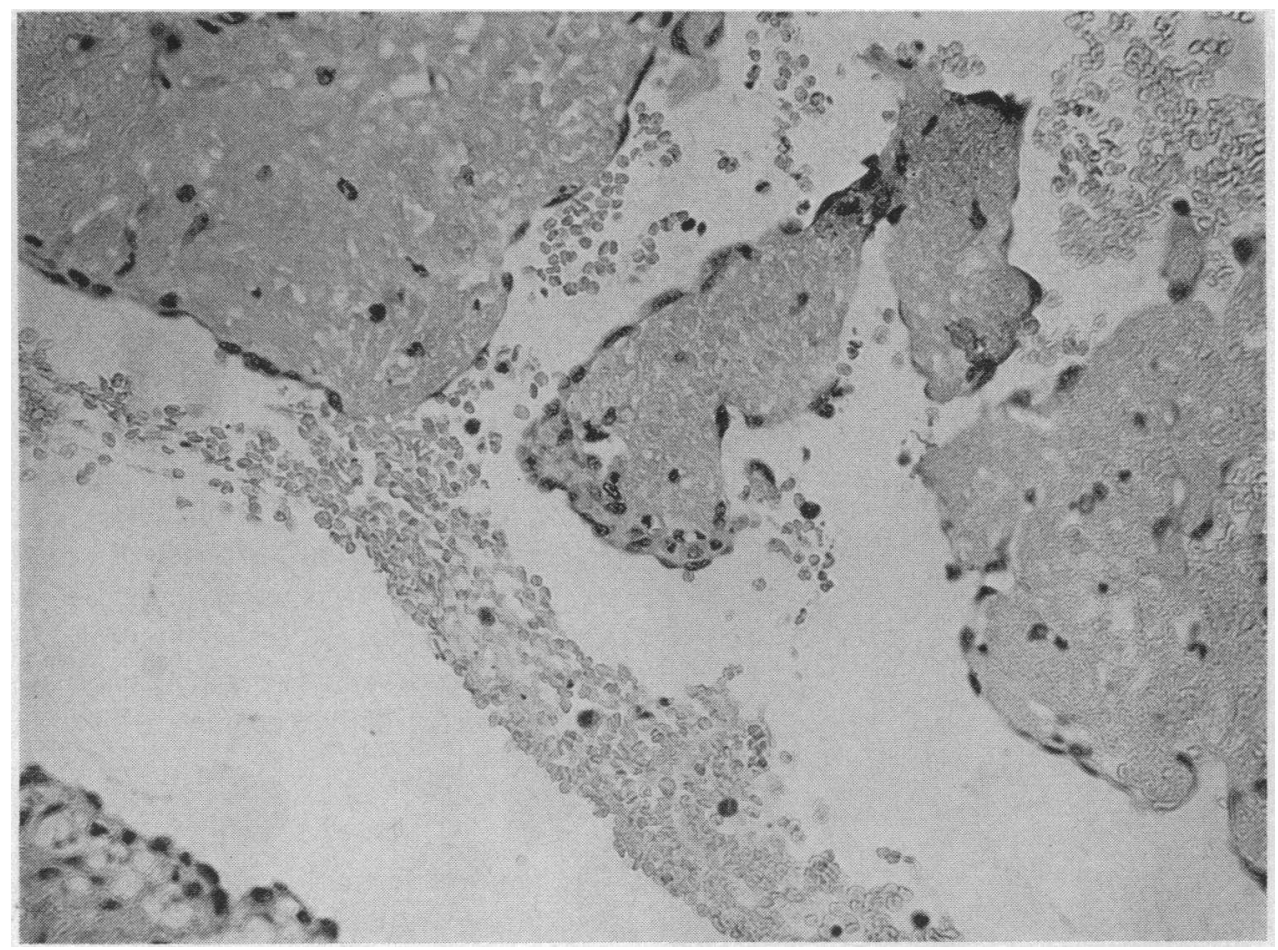

Fig. 3. Three Day Old Clot with Endothelial Covering Over the Ends (Hematoxylin Eosin $420 \times$ )

level used, clots three days of age or older can not effectively be dissolved with human plasmin.

\section{Histopathologic findings}

The histological findings were not uniform throughout the length of a clot. Despite this it was possible to grade the clots histologically and it was found that the histological picture correlated closely with their known age. The most important factor in grading was the degree of endothelial and fibroblastic activity and subsequent vascularization and fibrosis. The ends of the clot and spaces where the clot did not adhere firmly to the vessel wall were largely covered with endothelium by the third day (Figure 3 ) and about this time a few fibroblasts were occasionally present in the substance of the clot (Figure 4). Vascular spaces were formed soon after this (Figure 5). By the tenth day all clots were fully vascularized and many were only partially attached to the vessel wall with blood flowing around them (Figure 6). A second factor in grading was the change in the clot itself from the early mixture of fibrin and red cells to a more homogeneous mass from which the red cells gradually disappeared. Van Giesen stain revealed no collagen formation before the tenth day.

It was not possible to distinguish the clots which had received plasmin therapy after three or more days of existence from the control clots of similar age. Where treatment was given earlier, the clot appeared to be less advanced than expected at the time of removal. In some vessels where complete disappearance of the original isotopically-labeled fibrin structure was indicated by measurement of radioactivity, histologic examination revealed the presence of relatively fresh nonlabeled clots. It seemed probable that lysis of the original clot had been followed by formation of a fresh thrombus. Control clots of the same age showed little decrease of radioactivity levels. A varying degree of inflammatory reaction was always present in the vessel wall as a result of the manipulations necessary in the daily measurement of radioactivity. This may have influenced the reformation of the clot after the initial lysis. Occasionally, organization appeared less advanced than expected in the presence of severe necrosis of the vessel wall. 


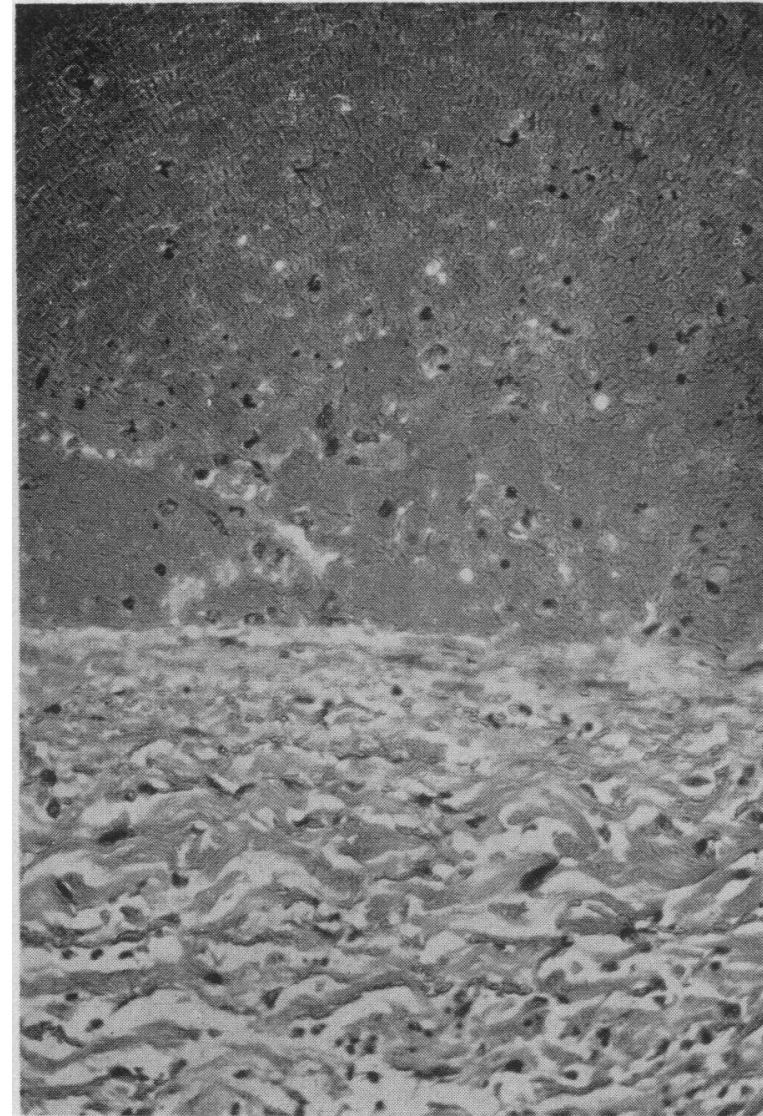

Fig. 4. Four Day Old Thrombus with Fibroblasts in Substance of Clot (Hematoxylin Fosin $420 \times$ )

\section{Biochemical findings}

In previous experiments $(1,2)$, human plasmin, prepared according to the method of Kline and activated with streptokinase in a dose of 30 plasmin units (Loomis) per $\mathrm{Kg}$. (4) had no effect on the fibrinogen and prothrombin level if given as a four hour intravenous infusion. In this study, acid extracts of Cohn's Fraction III activated with streptokinase were given by rapid intravenous injection. This resulted in a fall of fibrinogen levels as well as clotting index after the first injection. These changes did not usually occur during subsequent injections, but occasionally reappeared at the fifth daily injection.

\section{Hematologic findings}

Leukopenia was observed after each injection. No decrease of this change occurred during prolonged daily treatment. Leukopenia does not ap- pear to be due to the enzymatic effects of plasmin since plasminogen was shown to cause the same changes (2), and since Guest, Murphy, Bodnar, Ware, and Seegers (12) produced similar effects with inactivated bovine plasmin. Red blood cell counts, hematocrit and hemoglobin fluctuated within normal daily limits.

\section{Blood pressure}

In many dogs, carotid blood pressure was recorded during and after administration of plasmin. Rapid infusions of 30 plasmin units (Loomis) per $\mathrm{Kg}$. produced a small decrease of blood pressure ( 7 to $10 \mathrm{~mm}$. $\mathrm{Hg}$ ) which returned to normal within 10 minutes. In previous studies (2), a gradual decrease in the hypotensive activity was noticed during daily treatment with plasmin.

\section{DISCUSSION}

It appears from this and previous studies (2) that during daily human plasmin treatment, resistance gradually develops toward its fibrinolytic, hypotensive. fibrinogenopenic and prothrombinopenic, but not its leukopenic effects.

Resistance to lysis is apparent from the observation that most of the fibrinolytic effect is obtained within 24 hours after the first injection of plasmin, the remaining portions of the clot exhibiting less and less lysis during the subsequent days of treatment (2). It was found that the ability of plasmin at a dose level of 30 plasmin units (Loomis) per $\mathrm{Kg}$. to dissolve a clot is closely correlated with the degree of organization of the clot; the older the clot, the less lysis. It may be significant that even by the third day, most of the free edges of the clot were covered with a layer of endothelial cells (see Figure 3). No extensive histochemical study was made of the changes in the fibrin mass but collagen was not demonstrated before the tenth day. Williams (13), in a detailed study of the organization of artificially produced arterial clots, noted rapid endothelialization, occurring in the first 48 hours. He demonstrated a change in the staining reaction accompanying condensation of the fibrin after four days. Collagen appeared in the second week. It seems probable that the presence of endothelial cells may act as a physical barrier between the plasmin and the 


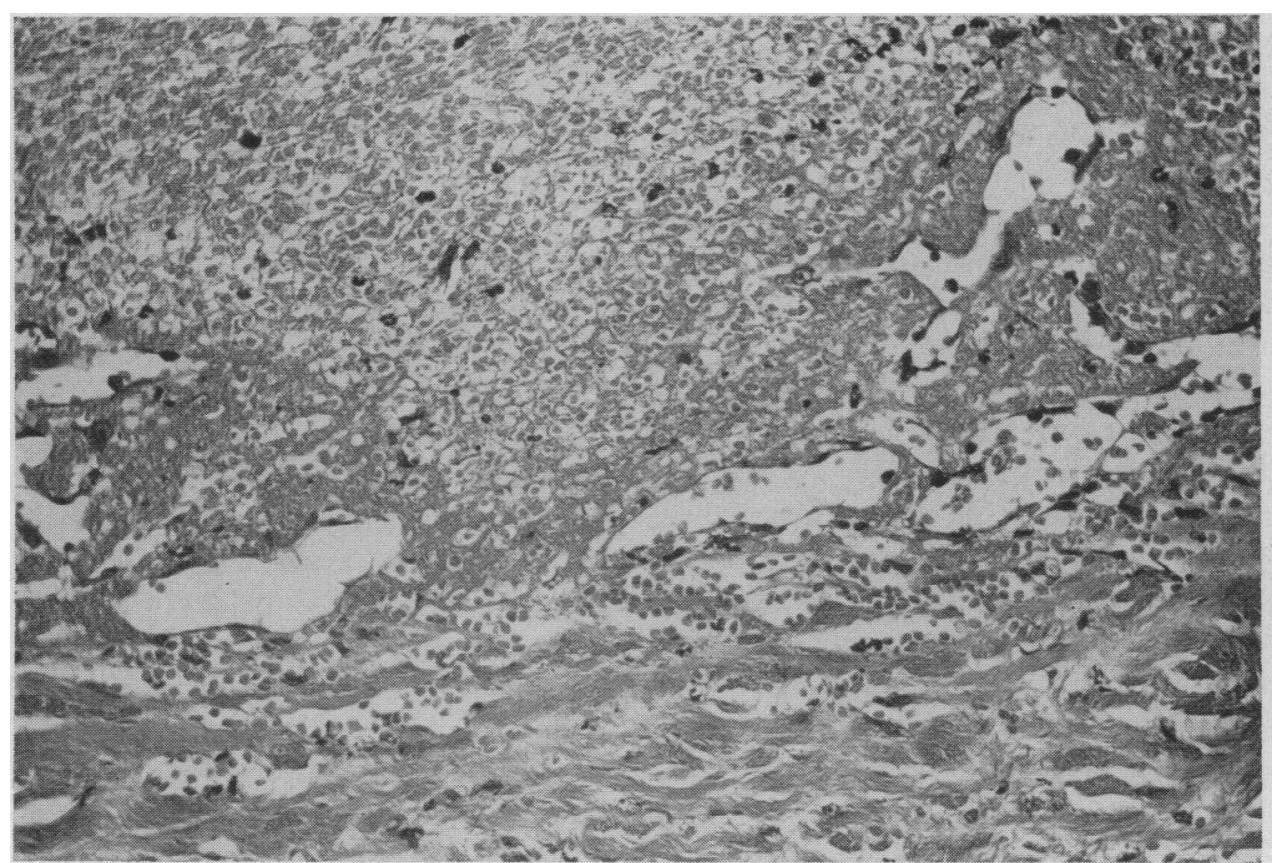

Fig. 5. Five Day Old Clot, Showing Vascularization (Hematoxylin Eosin $420 \times$ )

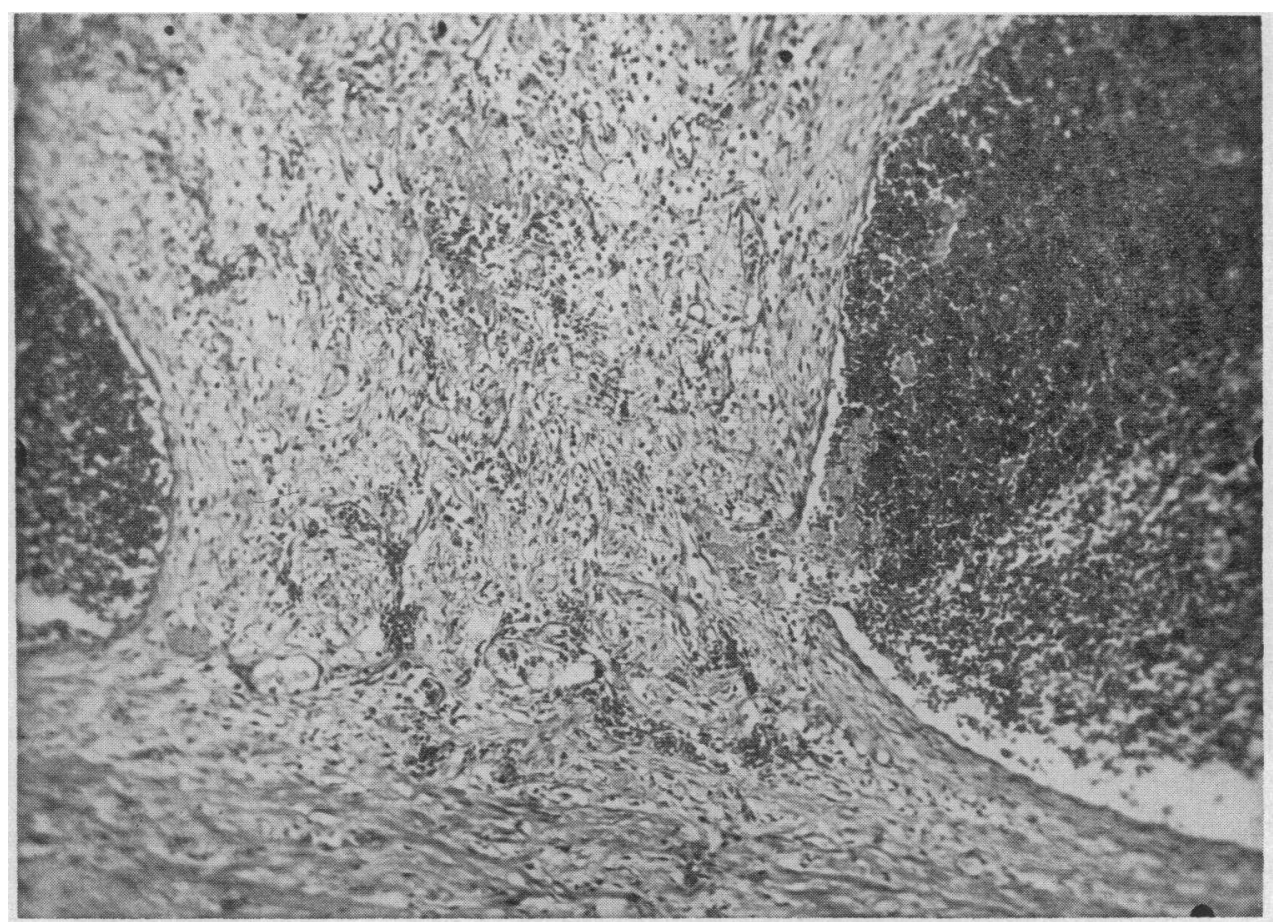

Fig. 6. Ten Day Old Control Clot with Extensive Vascularization and Partial Detachment from the Vessel Wall (Hematoxylin Eosin $200 \times$ )

Note fresh blood flowing through vessel between clot and wall. 
fibrin clot. In addition, increased density of the fibrin may also impair the effect of plasmin.

The above factors may explain resistance to lysis, but are certainly inadequate to account for the resistance toward biochemical and hypotensive changes. A study of changes in antiplasmin levels and antiplasmin antibody formation is presently under way. Results to date indicate that although chronic treatment with plasmin will induce fluctuations in these factors, they are insufficient to explain the degree of resistance encountered.

From the therapeutic point of view, it appears that acute thrombo-embolic disorders present better indications for plasmin therapy than chronic conditions. Payling-Wright (14) has suggested the possibility that cortisone given simultaneously with anticoagulants may delay organization of clots and increase the rate of recanalization of thrombosed vessels in rabbits. On the other hand, there are indications that cortisone may increase antiplasmin production or activity thus decreasing normal fibrinolytic activity of the blood (15).

After successful lysis of acute clots, reformation was observed in spite of continued treatment with plasmin. The experimental procedure called for opening the wound daily and inserting a lead shield and scintillation counter in the area. This obviously resulted in some degree of trauma and represented a possible source of infection despite daily administration of antibiotics. In addition, inflammatory reactions might have contributed to thrombus formation. These new clots were nonradioactive and were detected only upon autopsy. The fact that they did not lyse during daily treatment with plasmin (although they were fresh, unorganized clots) may be an expression of the general resistance developed toward plasmin as discussed above. Under most clinical conditions, stimuli for clot reformation are undoubtedly less than in the experimental arrangement reported here. The possibility of clot reformation after successful fibrinolytic therapy should be considered, particularly if a) the conditions originally responsible for clot formation still persist, and b).the temporary presence of the clot caused additional injury to the vessel wall.

\section{CONCLUSIONS}

Venous clots were produced with I $^{131}$-labeled fibrinogen in dogs at various time intervals. Five daily treatments with 30 plasmin units (Loomis) per $\mathrm{Kg}$. of human plasmin completely lysed clots less than three days old, but did not affect those older than three days. Histologic examination revealed that three days after formation, the clot is already largely separated from the blood stream by a layer of endothelial cells which may prevent plasmin from reaching the clot. Increased clot density may also be a factor in the clot becoming resistant to treatment.

In some instances, although the original radioactive clot completely disappeared, reformation of new, nonradioactive thrombi took place. Trauma might have been a major cause of this phenomenon. Reformation of the clot occurred in spite of daily administrations of plasmin. This may be related to the developing resistance of the animal to this agent as shown by decrease in changes of the fibrinogen levels, clotting index and blood pressure response $(2,4,16)$.

On the basis of these findings, it seems that better therapeutic results may be expected from intravenous treatment with plasmin in acute rather than chronic thromboembolic disorders. In the latter, results would appear to depend on the degree of organization of the clot.

\section{ACKNOWLEDGMENT}

This study was suggested by Dr. George F. Moore, Director, Roswell Park Memorial Institute, for whose advice the authors wish to express their gratitude. The assistance of J. W. Byron and Donna Halloran is gratefully acknowledged.

\section{REFERENCES}

1. Ambrus, J. L., Back, N., Mihalyi, E., and Ambrus, C. M. Quantitative method for the in vivo testing of fibrinolytic agents: Effect of intravenous trypsin on radioactive thrombi and emboli. Circulat. Res. 1956, 4, 430.

2. Back, N., Ambrus, J. L., Goldstein, S., and Harrisson, J. W. E. In vivo fibrinolytic activity and pharmacology of various plasmin (fibrinolysin) preparations. Circulat. Res. 1956, 4, 440.

3. Ambrus, J. L., Back, N., Goldstein, S., Ambrus, C. M., and Harrisson, J. W. E. In vivo fibrinolytic effect of various proteolytic enzymes; quantitative tests employing iodine ${ }^{131}$-labeled clots. In press.

4. Ambrus, J. L., Ambrus, C. M., Back, N., Sokal, J. E.,, and Collins, G. L. Clinical and experimental studies on fibrinolytic enzymes in Ann. N. Y. Acad. Sci. 1957, 68, 97. 
5. Loomis, E. C., George, C., Jr., and Ryder, A. Fibrinolysin: Nomenclature, unit, assay, preparation and properties. Arch. Biochem. 1947, 12, 1.

6. Remmert, L. F., and Cohen, P. P. Partial purification and properties of a proteolytic enzyme of human serum. J. biol. Chem. 1949, 181, 431.

7. Kline, D. L. Studies on the purification and activation of plasminogen (fibrinolysin). Yale J. Biol. Med. 1954, 26, 365.

8. Sherry, S., Titchener, A., Gottesman, L., Wasserman, P., and Troll, W. The enzymatic dissolution of experimental arterial thrombi in the dog by trypsin, chymotrypsin and plasminogen activators. J. clin. Invest. 1954, 33, 1303.

9. Mullertz, S., and Lassen, N. An activator system in blood indispensable for formation of plasmin by streptokinase. Proc. Soc. exp. Biol. (N. Y.) 1953, 82, 264.

10. Sherry, S. The fibrinolytic activity of streptokinase activated human plasmin. J. clin. Invest. 1954, 33, . 1054.

11. Kline, D. L., and Fishman, J. B. Plasmin-the hu- moral protease in Ann. N. Y. Acad. Sci. 1957, 68. 25.

12. Guest, M. M., Murphy, R. C., Bodnar, S. R., Ware, A. G., and Seegers, W. H. Physiological effects of a plasma protein: Blood pressure, leucocyte concentration, smooth and cardiac muscle activity. Amer. J. Physiol. 1947, 150, 471.

13. Williams, G. Experimental arterial thrombosis. J. Path. Bact. 1955, 69, 199.

14. Payling-Wright, $H$. Factors influencing the recanalisation of experimentally thrombosed blood vessels in Proc. First International Conference on Thrombosis and Embolism. Basel, Benno Schwabe \& Co., 1954, p. 565.

15. Stefanini, M., and Gendel, B. R. Influence of fibrinolysin on survival "in vivo" of various coagulation factors and favorable effect of cortisone in "fibrinolytic purpura." Clin. Res. Proc. 1953, 1, 5.

16. Back, N., and Ambrus, J. L. Mechanism of acquired resistance to plasmin (fibrinolysin). Changes in anti-plasmin levels during intravenous plasmin therapy. J. Pharmacol. exp. Therap. 1957, 119, 130. 